\title{
Prediction of mixture vapor-liquid equilibrium from the combined use of Peng-Robinson equation of state and COSMO-SAC activity coefficient model through the Wong-Sandler mixing rule
}

\author{
Ming-Tsung Lee, Shiang-Tai Lin* \\ Department of Chemical Engineering, National Taiwan University, Taipei, Taiwan 10617, Taiwan \\ Received 24 December 2006; received in revised form 9 February 2007; accepted 12 February 2007 \\ Available online 16 February 2007
}

\begin{abstract}
In this work we examined the prediction of vapor-liquid equilibria (VLE) of mixtures from the combined use of the Peng-Robinson equation of state (PR EOS) and the COSMO-SAC liquid activity coefficient model (LM). Based on the results of quantum mechanical calculations, it has been shown that the COSMO-SAC model is capable of predicting VLE of mixtures away from the critical point of any constituent component. Following the Wong-Sandler mixing rule, we found that the combined model is capable of predicting the VLE of binary mixtures, including alkane and alkane, alkane and alcohol, alkane and ketone, and alcohol and water, over a wide range of temperature (183.15-623.15 K) and pressure (0.1-19 MPa). Furthermore, it is found that the accuracy can be greatly improved when the Stavermann-Guggenheim combinatorial term in the COSMO-SAC model is ignored. The average error in both the pressure and vapor phase composition from the latter approach, denoted as PR + WS + COSMOSAC ${ }^{\text {res }}$, is lowered by more than $50 \%$ compared to that from the PR EOS with the van der Waals one fluid mixing rule $(\mathrm{PR}+\mathrm{VDW})$. Our results show that PR + WS + COSMOSAC ${ }^{\text {res }}$ is a promising approach for mixture VLE predictions over a large range of conditions. (C) 2007 Elsevier B.V. All rights reserved.
\end{abstract}

Keywords: Peng-Robinson equation of state; COSMO-SAC model; Wong-Sandler mixing rule; Vapor-liquid equilibrium prediction

\section{Introduction}

Thermodynamic models providing information on how a chemical species distributes among different phases in a system at various conditions is of fundamental importance in chemical processes and environmental engineering [1,2]. One important class of thermodynamics models, known as the PVT equations of state (EOS), focuses on the interrelationship between pressure, volume, and temperature. Such a type of models, e.g. the Peng-Robinson (PR) [3] or Soave-Redlich-Kwong (SRK) [4] EOS, is generally highly accurate for pure fluids over a wide range of conditions; however, their accuracy tends to deteriorate for mixture fluids, especially for nonideal systems, if the common van der Waals (VDW) one fluid mixing rule is employed. Another class of models, known as the liquid activity coefficient models (LM), focuses on the description of the excess Gibbs

\footnotetext{
* Corresponding author. Tel.: +88623366 1369; fax: +886223623040.

E-mail address: stlin@ntu.edu.tw (S.-T. Lin).
}

free energy of an incompressible liquid, from which the species activity coefficient is readily obtained. These models, such as the NRTL [5], Wilson [6], and UNIQUAC [7], are accurate for liquid mixtures but are not applicable in the vapor phase nor to pure substances.

For highly nonideal mixtures, efforts have been made to develop advanced mixing rules for use in the PVT EOS [8-22]. Among them, one appealing idea initiated by Huron and Vidal [11] was to incorporate the LM into PVT EOS so that the EOS can behave like a LM in the limit of infinite pressure. Although such Huron-Vidal (HV) mixing rule has led to many success in the description of complicated mixture phase behavior, it was pointed out [15] that the HV mixing rule does not satisfy the requirement from statistical mechanical theory that, in the lowdensity limit, the second virial coefficient be a quadric function of composition. More recently, Wong-Sandler (WS) [15] proposed a new type of mixing rule that ensures the quadric form of the second virial coefficient at all densities while retaining the original idea that the EOS behaves like a LM in the high pressure limit. 
In the present work, we intend to demonstrate that, following the WS mixing rule, the cubic type EOS can be made predictive for mixtures, i.e., without the need of any adjustable binary interaction parameter, when a predictive LM is used. Of special interest here is the COSMO-type LM originally proposed by Klamt [23] and later refined by Lin and Sandler [24] to ensure the thermodynamic consistency. This type of LM requires input only from the results of quantum mechanical calculations and is particularly useful when no experimental data is available. There have been many successful examples [14,25,26] in combing an EOS and LM through the WS mixing rule. However, most of them leave the binary interaction parameters in the EOS and/or LM as adjustable parameters.

There have been a few attempts in combining an EOS with a predictive LM; however, all of them took an "indirect" approach. For example, Constantinescu et al. [27] fitted the binary interaction parameters in the UNIQUAC model to the infinite dilution activity coefficient predicted from the COSMO-type models. The UNIQUAC model is then combined with the SRK EOS through the HV mixing rule for prediction of high pressure VLE for water-organic mixtures. Taking a similar approach but used activity coefficient at intermediate concentrations, Shimoyama et al. [28] reported successful VLE and/or LLE predictions for mixtures containing water, hydrocarbons and alcohols at high pressures. Our work here is different from these previous works in several aspects. First, we apply the predictive COSMO-type LM (the COSMO-SAC model [24] in our case) directly without the use of a second LM (e.g. UNIQUAC). The advantage is that no interpolation/extrapolation is necessary. In addition, we choose to use the Wong-Sandler mixing rule as it correctly reproduces the quadratic composition dependence at low-density limit. Finally, we have used the PR EOS in this work. While successful VLE predictions were obtained as in the previous works, the use of a predictive LM in a theoretically based mixing rule allows for a better examination of the ingredients that go into this composite model. Furthermore while all the models have a clear physical meaning, it is possible to understand why such an approach should work and/or fail under different circumstances.

\section{Theory}

The starting point for phase equilibrium calculations is the equality of fugacity in every phase for all species in a system. For example, when a vapor and a liquid phase are in equilibrium at some temperature $T$ and pressure $P$, we have:

$\bar{f}_{i}^{\mathrm{L}}\left(T, P, \underline{x}^{\mathrm{L}}\right)=\bar{f}_{i}^{\mathrm{V}}\left(T, P, \underline{x}^{\mathrm{V}}\right)$

where the superscript $\mathrm{L}$ or $\mathrm{V}$ denotes the vapor or liquid phase, $\bar{f}_{i}$ the fugacity of species $i$, and $x$ denotes the molar composition of the phase. The fugacity is determined according to [1]:

$$
\begin{aligned}
\ln \frac{\bar{f}_{i}(T, P, \underline{x})}{x_{i} P} & =\frac{1}{R T} \int_{\infty}^{Z R T / P}\left(\frac{R T}{\underline{V}}-N\left(\frac{\partial P}{\partial N_{i}}\right)_{T, V, n_{j \neq i}}\right) \mathrm{d} \underline{V} \\
& -\ln Z
\end{aligned}
$$

where $N_{i}$ is the number of moles of species, $N=\Sigma N_{i}$ the total number of moles, and $Z=P V / R T$ is the compressibility factor. A PVT EOS is needed for the evaluation of the integral in Eq. (2). Assuming that the PVT relations of mixture fluids obey the Peng-Robinson EOS, we have:

$P=\frac{R T}{\underline{V}-b(\underline{x})}-\frac{a(T, \underline{x})}{\underline{V}^{2}+2 b(\underline{x}) \underline{V}-b(\underline{x})^{2}}$

where $a(T, \underline{x})$ and $b(\underline{x})$ are the pseudo one fluid parameters for the mixture and can be determined from a mixing rule.

In the van der Waals one fluid mixing rule, mixture parameters $a$ and $b$ are determined from its constituent components according to the following two conditions:

$a(T, \underline{x})=\sum_{i} \sum_{j} x_{i} x_{j} a_{i j}$

$b(\underline{x})=\sum_{i} x_{i} b_{i}$

with $a_{i j}$ determined from the combining rule:

$a_{i j}=\sqrt{a_{i} a_{j}}\left(1-k_{i j}\right)$

where $k_{i j}$ is the binary interaction parameter that can be obtained only from fitting to experimental data. The parameters $a_{i}$ and $b_{i}$ of pure component $i$ are determined from the critical properties:

$a_{i}(T)=0.45724 \frac{R^{2} T_{\mathrm{c}, i}^{2}}{P_{\mathrm{c}, i}} \alpha_{i}(T)$

$b_{i}=0.07780 \frac{R T_{\mathrm{c}, i}}{P_{\mathrm{c}, i}}$

with $\sqrt{\alpha_{i}(T)}=1+\kappa_{i}\left(1-\sqrt{T / T_{\mathrm{c}, i}}\right)$ and $\kappa_{i}=0.37464+$ $1.54226 \omega_{i}-0.26992 \omega_{i}^{2}$ where $\omega_{i}$ is the acentric factor of species $i$. Using the PR EOS and VDW mixing rule, Eq. (2) becomes:

$$
\begin{aligned}
\ln \frac{\bar{f}_{i}(T, P, \underline{x})}{x_{i} P}= & \frac{b_{i}}{b}(Z-1)-\ln \left(Z-\frac{b P}{R T}\right)-\frac{a}{2 \sqrt{2} b R T} \\
& \times\left(\frac{2 \sum_{j} x_{j} a_{i j}}{a}-\frac{b_{i}}{b}\right) \ln \frac{Z R T+(1+\sqrt{2}) b P}{Z R T+(1-\sqrt{2}) b P}
\end{aligned}
$$

When the Wong-Sandler mixing rule is used, mixture parameters $a$ and $b$ are determined as:

$a=R T \frac{Q D}{1-D}$

$b=\frac{Q}{1-D}$

with $Q=\sum_{i} \sum_{j} x_{i} x_{j}(b-(a / R T))_{i j}, \quad D=\sum_{i} x_{i}\left(a_{i} / b_{i} R T\right)+$ $\left(G^{\mathrm{ex}} / C R T\right)$, and $C=-(\ln (1+\sqrt{2}) / \sqrt{2})$. The term $(\bar{b}-(a / R T))_{i j}$ is determined with the following combining 
rule:

$$
\left(b-\frac{a}{R T}\right)_{i j}=\frac{1}{2}\left[\left(b_{i}-\frac{a_{i}}{R T}\right)+\left(b_{j}-\frac{a_{j}}{R T}\right)\right]\left(1-k_{i j}\right)
$$

In order to make the model predictive, we have set the binary interaction parameters $k_{i j}$ in Eqs. (6) and (11) to zero in our work. Using the PR EOS and WS mixing rule, Eq. (2) becomes:

$$
\begin{aligned}
\ln \frac{\bar{f}_{i}(T, P, \underline{x})}{x_{i} P} & \frac{1}{b}\left(\frac{\partial N b}{\partial N_{i}}\right)_{T, N_{j \neq i}}(Z-1)-\ln \left(Z-\frac{b P}{R T}\right) \\
& -\frac{a}{2 \sqrt{2} b R T}\left(\frac{1}{N a}\left(\frac{\partial N^{2} a}{\partial N_{i}}\right)_{T, N_{j \neq i}}-\frac{1}{b}\left(\frac{\partial N b}{\partial N_{i}}\right)_{T, N_{j \neq i}}\right) \\
& \ln \frac{Z R T+(1+\sqrt{2}) b P}{Z R T+(1-\sqrt{2}) b P}
\end{aligned}
$$

where

$$
\begin{aligned}
&\left(\frac{\partial N b}{\partial N_{i}}\right)_{T, N_{j \neq i}}= \frac{1}{1-D} \frac{1}{N}\left(\frac{\partial N^{2} Q}{\partial N_{i}}\right)_{T, N_{j \neq i}}-\frac{Q}{(1-D)^{2}} \\
& \times\left[1-\left(\frac{\partial N D}{\partial N_{i}}\right)_{T, N_{j \neq i}}\right] \\
& \frac{1}{N}\left(\frac{\partial N^{2} a}{\partial N_{i}}\right)_{T, N_{j \neq i}}=\operatorname{RTD}\left(\frac{\partial N b}{\partial N_{i}}\right)_{T, N_{j \neq i}}+R T b\left(\frac{\partial N D}{\partial N_{i}}\right)_{T, N_{j \neq i}}
\end{aligned}
$$

with

$$
\begin{aligned}
& \frac{1}{N}\left(\frac{\partial N^{2} Q}{\partial N_{i}}\right)_{T, N_{j \neq i}}=2 \sum_{j} x_{j}\left(b-\frac{a}{R T}\right)_{i j} \\
& \left(\frac{\partial N D}{\partial N_{i}}\right)_{T, N_{j \neq i}}=\frac{a_{i}}{b_{i} R T}+\frac{\ln \gamma_{i}}{C}
\end{aligned}
$$

The activity coefficient $\gamma_{i}$ needed in the WS mixing rule (Eq. (17)) can be determined from the COSMO-SAC model [24], in which the activity coefficient $\gamma_{i}$ of species $i$ is calculated from the sum of the residual and combinatorial contributions:

$\ln \gamma_{i}=\ln \gamma_{i}^{\mathrm{res}}+\ln \gamma_{i}^{\mathrm{comb}}$

The residual part of $\gamma$ is calculated from a consideration of molecular solvation in a perfect conductor. The distribution of screening charges on the molecular surface, called the sigma profile $p(\sigma)$, is first determined from quantum mechanical calculations. The molecular interactions in the liquid phase are assumed to be the sum of contributions of surface segment interactions through the screening charges. With these assumptions, the $\gamma_{i}^{\text {res }}$ takes the following form:

$$
\ln \gamma_{i}^{\mathrm{res}}=n_{i} \sum_{\sigma_{m}} p_{i}\left(\sigma_{m}\right) \ln \left[\Gamma_{\mathrm{S}}\left(\sigma_{m}\right)-\Gamma_{i}\left(\sigma_{m}\right)\right]
$$

where $n_{i}$ is the number of surface segments contained in species $i, \Gamma_{\mathrm{S}}(\sigma)$ the activity coefficient of segment $i$ (whose screening charge density is $\sigma$ ) in solution $\mathrm{S}$ (for which the probability of finding a segment of charge density $\sigma$ be denoted $\left.p_{\mathrm{S}}(\sigma)\right)$ :

$\ln \Gamma_{\mathrm{S}}\left(\sigma_{m}\right)=-\ln \left\{\sum_{\sigma_{n}} p_{\mathrm{S}}\left(\sigma_{n}\right) \Gamma_{\mathrm{S}}\left(\sigma_{n}\right) \exp \left[\frac{-\Delta W\left(\sigma_{m}, \sigma_{n}\right)}{R T}\right]\right\}$

where $\Delta W\left(\sigma_{m}, \sigma_{n}\right)$ is the electrostatic interaction between two segments of charge density $\sigma_{m}$ and $\sigma_{n}$. The Staverman-Guggenheim (SG) model is used for the combinatorial term:

$\ln \gamma_{i}^{\mathrm{comb}}=\ln \frac{\phi_{i}}{x_{i}}+\frac{z}{2} q_{i} \ln \frac{\theta_{i}}{\phi_{i}}+l_{i}-\frac{\phi_{i}}{x_{i}} \sum_{j} x_{j} l_{j}$

with the surface area fraction $\theta_{i}=x_{i} q_{i} / \sum_{j} x_{j} q_{j}$, volume fraction $\phi_{i}=x_{i} r_{i} / \sum_{j} x_{j} r_{j}$, and $l_{i}=(z / 2)\left(r_{i}-q_{i}\right)-\left(r_{i}-1\right)$, where $r_{i}$ and $q_{i}$ are the normalized volume and surface area parameters for $i$; $z$ is the coordination number (taken to be 10). We refer the LM to COSMOSAC ${ }^{\text {res }}$ for cases where the SG combinatorial term is ignored.

\section{Computation details}

The vapor-liquid equilibrium (VLE) phase diagrams of 12 binary mixtures covering a wide range of temperature (183.15-623.15 K) and pressure (0.1-19 MPa) are examined. The interaction between the species in the chosen systems ranges from weakly interacting (alkane and alkane), to medium interacting (alkane and ketone, alkane and alcohol), and to strongly interacting (alcohol and water). A list of the systems studied in this work is given in Table 1 .

For each system, the VLE phase diagram is predicted using three methods: PR + VDW (Eq. (9)), PR + WS + COSMOSAC (Eqs. (13)-(21)), and $\mathrm{PR}+\mathrm{WS}+\mathrm{COSMOSAC}{ }^{\text {res }}$ (Eqs. (13)-(20)) [Note that the notation $\mathrm{A}+\mathrm{B}+\mathrm{C}$ used here indicates the EOS (A), mixing rule (B), and LM (C) used.] For a given liquid phase composition, the bubble point pressure calculation, as detailed by Sandler [1], is performed to obtain the system pressure and the vapor phase compositions. In order to make the models predictive, the binary interaction parameters $k_{i j}$ in the combining rules are set to zero.

If the WS mixing rule is used, the activity coefficient of each species must be provided at a given temperature and mixture composition. For each compound, the equilibrium molecular geometry is first determined by minimization of the molecular energy at $0 \mathrm{~K}$ using the quantum chemistry package $\mathrm{DMol}^{3}$ implemented in Cerius ${ }^{2}$ [29]. A solvation calculation in perfect conductor is then performed using the equilibrium geometry to obtain the surface screening charges on the compound. The detailed settings for $\mathrm{DMol}^{3}$ can be found elsewhere [24]. The activity coefficient is then calculated from Eqs. (12)-(15) using an in-house program. Note that for each species, the quantum mechanical part of this calculation, which may be 
Table 1

Comparison of absolute average error in equilibrium pressure and vapor phase compositions from different methods

\begin{tabular}{|c|c|c|c|c|c|c|c|}
\hline \multirow[t]{3}{*}{ Mixture } & \multicolumn{7}{|l|}{ Model } \\
\hline & \multicolumn{3}{|c|}{$\mathrm{PR}+\mathrm{VDW}$} & \multicolumn{2}{|c|}{$\mathrm{PR}+\mathrm{WS}+\mathrm{COSMOSAC}$} & \multicolumn{2}{|c|}{$\mathrm{PR}+\mathrm{WS}+\mathrm{COSMOSAC} \mathrm{C}^{\mathrm{res}}$} \\
\hline & $T(\mathrm{~K})$ & AARD $P(\%)$ & AARD $y_{1}(\%)$ & $\operatorname{AARD} P(\%)$ & AARD $y_{1}(\%)$ & AARD $P(\%)$ & AARD $y_{1}(\%)$ \\
\hline \multirow{2}{*}{$\begin{array}{l}\text { Pentane/hexane } \\
{[30]}\end{array}$} & 298.15 & 1.22 & 0.87 & 1.10 & 0.76 & 1.23 & 0.00 \\
\hline & 303.70 & 3.25 & 1.86 & 3.41 & 1.92 & 3.29 & 0.04 \\
\hline Hexane/octane [30] & 328.15 & 2.26 & 1.07 & 1.68 & 0.78 & 2.07 & 0.97 \\
\hline Octane/heptane [30] & 328.15 & 1.43 & 3.21 & 1.54 & 3.31 & 1.47 & 3.25 \\
\hline Methane/ethane [31] & 186.11 & 1.56 & 0.27 & 2.17 & 0.41 & 1.38 & 0.18 \\
\hline \multirow{5}{*}{$\begin{array}{l}\text { Propane/ethane } \\
\text { [32] }\end{array}$} & 255.37 & 0.83 & 1.42 & 0.92 & 1.60 & 0.84 & 1.43 \\
\hline & 283.15 & 1.03 & 0.57 & 1.25 & 0.77 & 1.04 & 0.58 \\
\hline & 310.93 & 0.99 & 0.35 & 0.86 & 0.43 & 0.99 & 0.34 \\
\hline & 344.26 & 1.06 & 3.83 & 1.14 & 3.68 & 1.06 & 3.83 \\
\hline & 366.48 & 0.64 & 13.45 & 0.60 & 16.13 & 0.64 & 13.44 \\
\hline \multirow{2}{*}{$\begin{array}{l}\text { Methane/hexane } \\
{[33,34]}\end{array}$} & 183.15 & 23.83 & 0.09 & 36.67 & 0.09 & 23.66 & 0.09 \\
\hline & 373.00 & 6.10 & 2.49 & 10.71 & 6.51 & 6.17 & 2.63 \\
\hline \multirow{4}{*}{$\begin{array}{l}\text { Pentane/1- } \\
\text { butanol } \\
{[35]}\end{array}$} & 468.15 & 16.22 & 2.67 & 6.21 & 7.35 & 4.65 & 5.37 \\
\hline & 483.15 & 12.88 & 4.91 & 6.89 & 7.26 & 7.00 & 6.56 \\
\hline & 498.15 & 8.54 & 6.75 & 3.06 & 14.91 & 3.34 & 13.54 \\
\hline & 513.15 & 7.06 & 5.87 & 3.31 & 3.62 & 3.29 & 3.64 \\
\hline \multirow{4}{*}{$\begin{array}{l}\text { Pentane/2- } \\
\text { butanol } \\
{[35]}\end{array}$} & 468.15 & 10.91 & 5.65 & 4.72 & 3.10 & 4.67 & 4.67 \\
\hline & 483.15 & 7.55 & 9.74 & 3.55 & 4.27 & 3.51 & 3.51 \\
\hline & 498.15 & 6.07 & 5.21 & 3.31 & 2.99 & 3.28 & 3.28 \\
\hline & 513.15 & 2.31 & 10.33 & 0.97 & 6.14 & 0.95 & 0.95 \\
\hline \multirow{3}{*}{$\begin{array}{l}\text { Pentane/ethanol } \\
\text { [36] }\end{array}$} & 422.60 & 19.27 & 16.12 & 12.82 & 11.03 & 11.92 & 10.20 \\
\hline & 465.40 & 16.65 & 6.19 & 11.84 & 5.02 & 10.88 & 4.56 \\
\hline & 500.00 & 7.39 & 14.06 & 3.55 & 14.04 & 3.27 & 13.27 \\
\hline Acetone/hexane [37] & 318.15 & 23.64 & 20.18 & 13.79 & 10.89 & 12.62 & 9.90 \\
\hline \multirow{7}{*}{$\begin{array}{l}\text { Ethanol/water } \\
\text { [38] }\end{array}$} & 423.15 & 31.20 & 15.83 & 10.40 & 13.21 & 3.75 & 6.19 \\
\hline & 473.15 & 17.31 & 126.50 & 9.66 & 13.64 & 3.05 & 3.89 \\
\hline & 523.15 & 15.33 & 89.72 & 7.92 & 12.69 & 2.17 & 2.58 \\
\hline & 548.15 & 12.28 & 54.16 & 6.71 & 11.79 & 1.45 & 1.69 \\
\hline & 573.15 & 8.27 & 56.27 & 4.98 & 16.28 & 1.28 & 4.96 \\
\hline & 598.15 & 5.29 & 71.17 & 2.67 & 8.78 & 0.47 & 3.56 \\
\hline & 623.15 & 1.82 & 30.27 & 1.62 & 10.51 & 0.88 & 3.04 \\
\hline \multirow{5}{*}{$\begin{array}{l}2- \\
\text { Propanol/water } \\
{[38]}\end{array}$} & 423.15 & - & - & 13.98 & 2.72 & 7.85 & 12.78 \\
\hline & 473.15 & - & - & 12.12 & 15.28 & 5.96 & 6.12 \\
\hline & 523.15 & - & - & 8.30 & 17.93 & 2.20 & 6.29 \\
\hline & 548.15 & 22.45 & 33.82 & 8.08 & 13.42 & 1.60 & 4.95 \\
\hline & 573.15 & 13.52 & 35.01 & 3.10 & 21.12 & 1.19 & 7.45 \\
\hline \multicolumn{2}{|l|}{ Overall AARD } & 8.85 & 18.74 & 6.27 & 7.90 & 4.03 & 4.71 \\
\hline
\end{tabular}

time consuming, only has to be done once regardless of the temperature and the composition of mixture needed.

The absolute average error in pressure and vapor phase composition from the calculation is determined as:

AARD $P=\frac{1}{n} \sum_{i}^{n}\left|\frac{P^{\exp }-P^{\text {cal }}}{P^{\exp }}\right|$

and

$$
\operatorname{AARD} y_{i}=\frac{1}{n} \sum_{i}^{n}\left|\frac{y_{i}^{\exp }-y_{i}^{\mathrm{cal}}}{y_{i}^{\exp }}\right|
$$

where $n$ is the number of data point, superscripts exp and cal denote the values from experiment and our calculation, respectively.

\section{Results and discussions}

Table 1 summarizes the errors in VLE prediction for 12 binary mixtures at different temperatures. The absolute average errors in the calculated pressure are 9, 6, and $4 \%$ from PR + VDW, PR + WS + COSMOSAC, and $\mathrm{PR}+\mathrm{WS}+\mathrm{COSMOSAC}{ }^{\text {res }}$, respectively. The corresponding errors in the vapor phase composition are 19, 8 , and $5 \%$ from the three methods. The PR + WS + COSMOSAC ${ }^{\text {res }}$ approach gives 


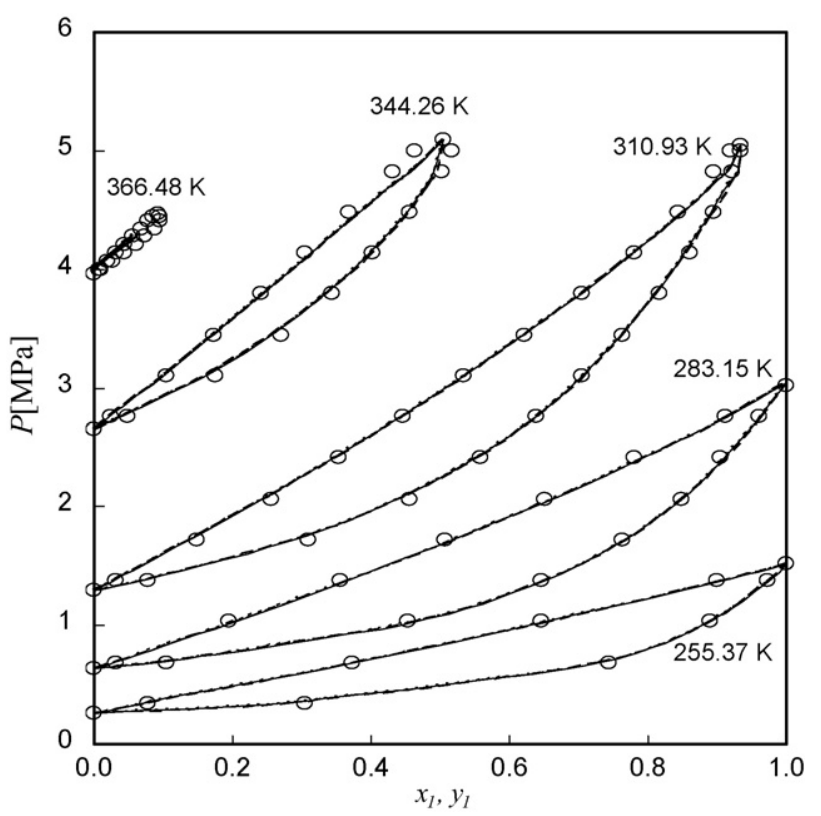

Fig. 1. Vapor-liquid equilibrium pressures and phase compositions for propane (1)/ethane (2) mixtures at 255.37, 283.15, 310.93, 344.26 and $366.48 \mathrm{~K}$. Open circles represent experimental data. Solid lines are predictions from $\mathrm{PR}+\mathrm{WS}+\mathrm{COSMOSAC}{ }^{\text {res }}$, dashed lines from PR + WS + COSMOSAC, and dotted lines from PR + VDW.

the best predictions. The average error is lowered by more than $50 \%$ compared to that from PR + VDW, especially for highly nonideal systems. (Note that in some cases the PR + VDW method failed to converge in the bubble point calculations.)

For systems containing weakly interacting species, the performance of the three methods are similar. For example, the VLE predictions for propane/ethane mixtures from the three methods are indistinguishable in Fig. 1. However, when the component species are highly dissimilar in size, as in the

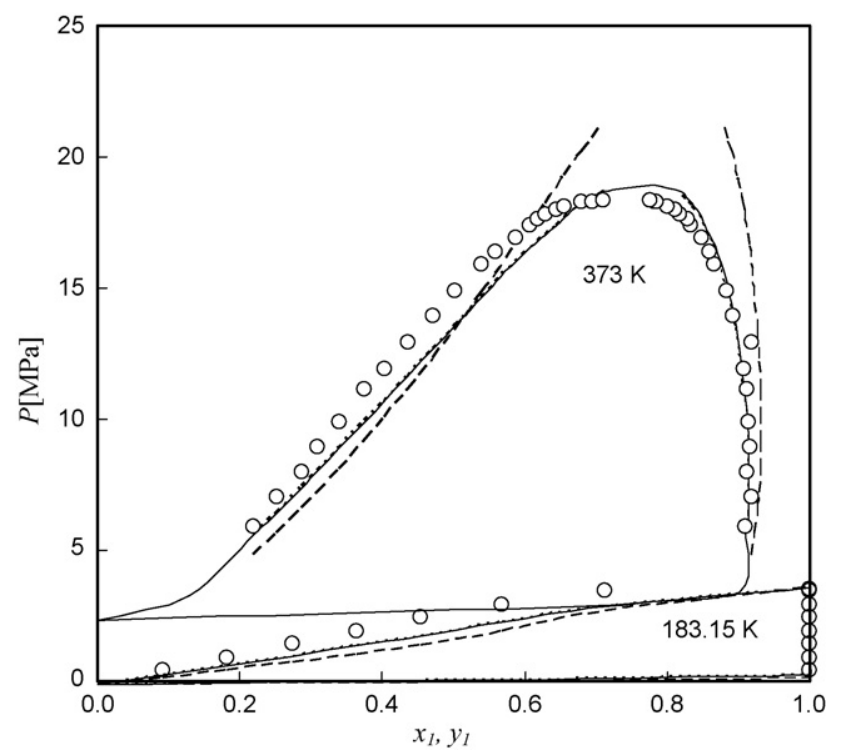

Fig. 2. Vapor-liquid equilibrium pressures and phase compositions for methane (1)/hexane (2) mixtures at 183.15 and $373 \mathrm{~K}$. The legends are the same as in Fig. 1.

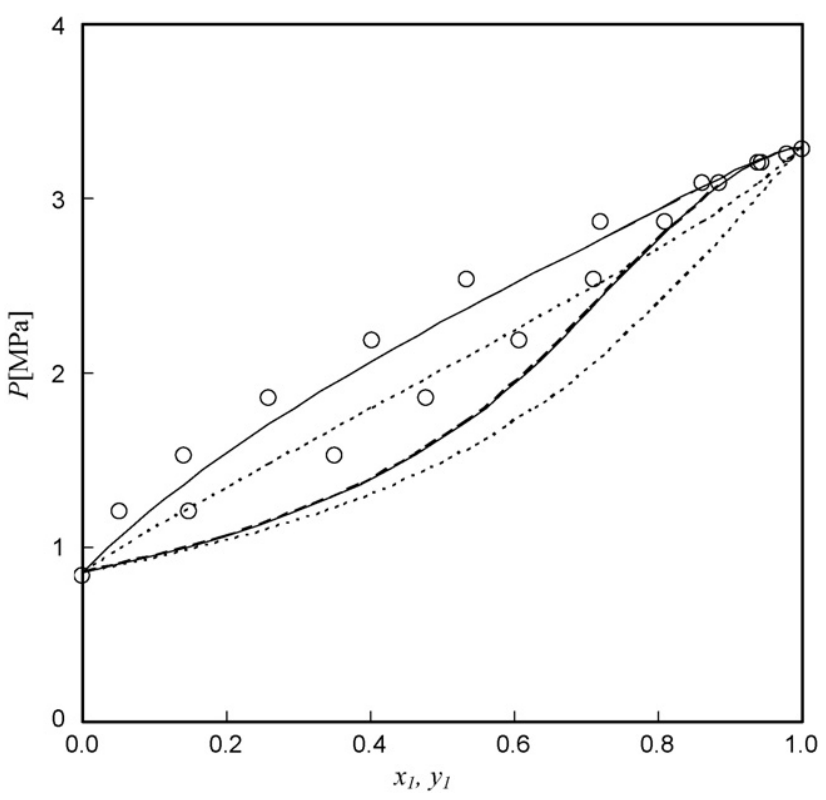

Fig. 3. Vapor-liquid equilibrium pressures and phase compositions for pentane (1)/1-butanol (2) mixtures at $468.15 \mathrm{~K}$. The legends are the same as in Fig. 1. Results from PR +WS +COSMOSAC (dashed line) and $\mathrm{PR}+\mathrm{WS}+\mathrm{COSMOSAC} \mathrm{C}^{\mathrm{res}}$ (solid line) are indistinguishable in this figure.

methane/hexane mixture shown in Fig. 2, the performance from $\mathrm{PR}+\mathrm{WS}+\mathrm{COSMOSAC}$ deteriorates, especially at high temperatures. Since for alkane-alkane systems the residual contribution to the activity coefficient (Eq. (19)) is nearly zero, the much higher critical pressure from PR + WS + COSMOSAC at $373 \mathrm{~K}$ indicates that the $\mathrm{SG}$ combinatorial term provides a too negative value of $G^{\text {ex }}$ for this system.

As the nonideality in the system increases, the superiority of using the more advanced WS mixing rule becomes clear. Figs. 3-5 illustrate the predictions for pentane/1-butanol, acetone/hexane, and 2-propanol/water systems, respectively. As can be seen, PR + VDW starts failing to predict the correct phase diagram (e.g. Figs. 4 and 5) even at low temperatures. The PR + WS + COSMOSAC method performs reasonably well at

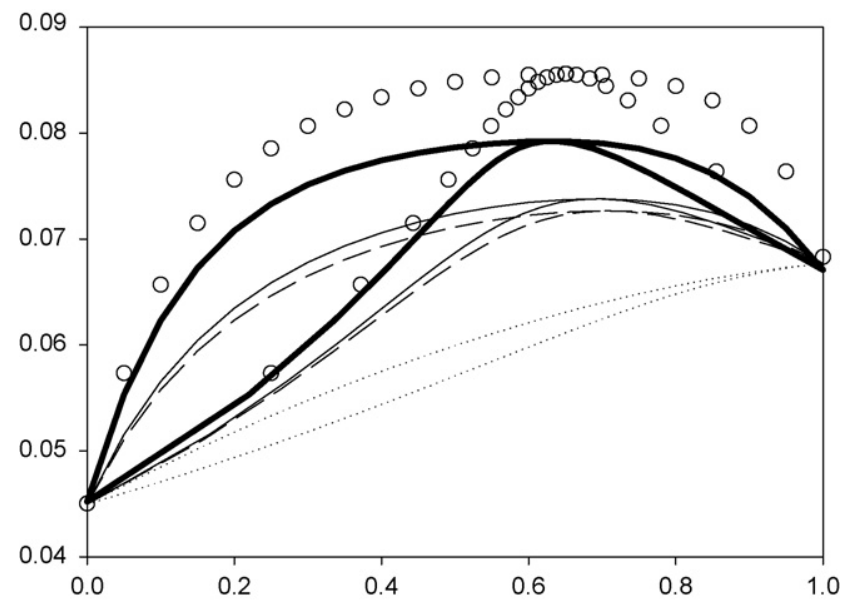

Fig. 4. Vapor-liquid equilibrium pressures and phase compositions for acetone (1)/hexane (2) mixtures at $318.15 \mathrm{~K}$. Bold solid lines are predictions from the COSMO-SAC model. Other legends are the same as in Fig. 1. 


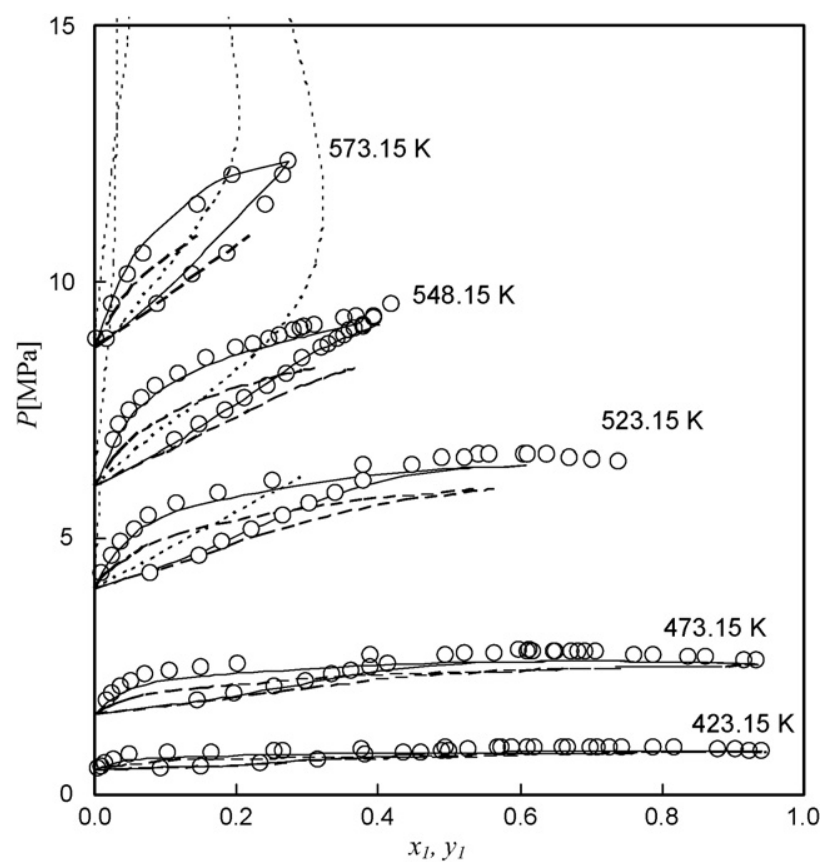

Fig. 5. Vapor-liquid equilibrium pressures and phase compositions for 2propanol (1)/water (2) mixtures at 423.15, 473.15, 523.15, 548.15 and $573.15 \mathrm{~K}$. The legends are the same as in Fig. 1.

low temperatures but starts to deviate from experiment at higher temperatures (e.g. Fig. 5). (The seemingly lowered AARD in pressure in Table 1 is a result of the higher pressures at high temperatures.) This is similar to the results observed in the alkane-alkene systems. In contrast, the behavior from $\mathrm{PR}+\mathrm{WS}+\mathrm{COSMOSAC} \mathrm{C}^{\text {res }}$ is very consistent for all temperatures.

The variation of predictive power in the three methods for systems of different degrees of nonideality and at different temperatures and pressure can be better elucidated from the excess Gibbs free energy. Fig. 6 shows the liquid phase $G^{\text {ex }}$ for the acetone-hexane mixture (VLE diagram in Fig. 4) at $318.15 \mathrm{~K}$.

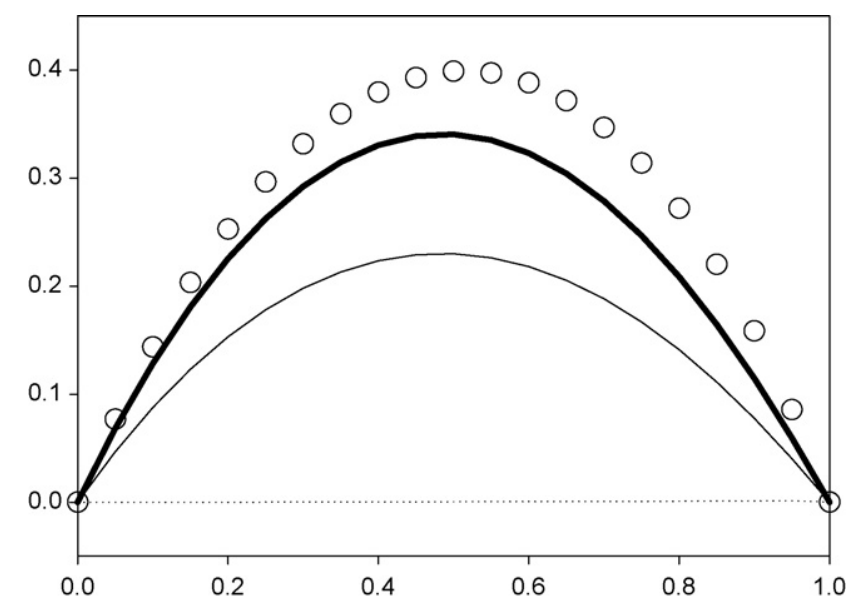

Fig. 6. The figure of normalized excess Gibbs free energy in liquid phase for acetone (1)/hexane (2) mixtures. The legends are the same as in Fig. 4. Results from $\mathrm{PR}+\mathrm{WS}+\mathrm{COSMOSAC}$ (dashed line) and PR + WS + COSMOSAC ${ }^{\text {res }}$ (solid line) are indistinguishable in this figure.
To compare, the predictions from the COSMO-SAC model alone (i.e., assuming ideal vapor phase) is also shown (bold sold curves). It is clear that PR + VDW failed to describe the azeotropic behavior in this system because the calculated $G^{\text {ex }}$ (dotted line) for the liquid phase are almost zero (i.e., the mixture is almost ideal). Though underestimated, the liquid phase $G^{\text {ex }}$ calculated from the COSMO-SAC is closest to experiment. As a consequence, COSMO-SAC alone gives the best VLE prediction for this system. When combined with PR EOS through the WS mixing rule (i.e., PR + WS + COSMOSAC), the value of calculated $G^{\text {ex }}$ is further reduced. Therefore, the predicted VLE is less accurate. The reduction in $G^{\mathrm{ex}}$ is a result of the nonzero excess volume and can be understood as follows. The excess Gibbs free energy (calculated from the EOS) $G_{\mathrm{EOS}}^{\mathrm{ex}}$ is the sum of excess Helmholtz free energy and the product of pressure and excess volume:

$G_{\mathrm{EOS}}^{\mathrm{ex}}(T, P, \underline{x})=\underline{A}_{\mathrm{EOS}}^{\mathrm{ex}}(T, P, \underline{x})+P \underline{V}_{\mathrm{EOS}}^{\mathrm{ex}}(T, P, \underline{x})$

Since in the WS mixing rule the excess Helmholtz free energy at liquid densities is assumed to be a weak function of pressure, i.e., $\underline{A}^{\mathrm{ex}}(T, P, \underline{x}) \approx \underline{A}^{\mathrm{ex}}(T, P \rightarrow \infty, \underline{x})$, and, furthermore, its value is set to the excess Gibbs free energy from LM, i.e., $A_{\mathrm{EOS}}^{\mathrm{ex}}(T, P \rightarrow$ $\infty, \underline{x})=G_{\mathrm{LM}}^{\mathrm{ex}}(T, \underline{x})$. As a consequence, Eq. (24) becomes:

$G_{\mathrm{EOS}}^{\mathrm{ex}}(T, P, \underline{x}) \approx G_{\mathrm{LM}}^{\mathrm{ex}}(T, \underline{x})+P \underline{V}_{\mathrm{EOS}}^{\mathrm{ex}}(T, P, \underline{x})$

Therefore, using an LM which is accurate at ambient conditions does not guarantee an equally accurate prediction of VLE at the same condition when it is combined with and EOS. Interestingly, since the SG combinatorial term contributes negatively to $G^{\text {ex }}$ (though the magnitude is small in this case), the prediction is slightly improved when it is ignored (i.e., $\mathrm{PR}+\mathrm{WS}+$ COSMOSAC ${ }^{\mathrm{res}}$ ).

To understand the temperature effects, Fig. 7 shows the liquid phase $G^{\text {ex }}$ for the 2-propanol-water mixture (VLE diagram

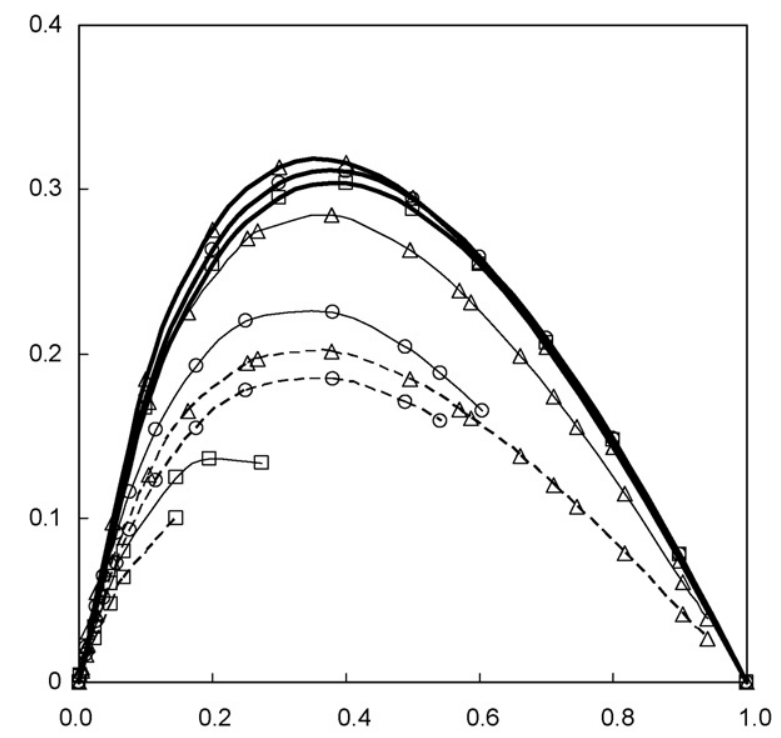

Fig. 7. The figure of normalized excess Gibbs free energy in liquid phase for 2-propanol (1)/water (2) mixtures under $423.15 \mathrm{~K}$ (open triangle), $523 \mathrm{~K}$ (open circle) and $573 \mathrm{~K}$ (open square). The legends are the same as in Fig. 5. 
in Fig. 5) at three temperatures 423.15, 523 and $573 \mathrm{~K}$. The values from COSMO-SAC model are shown as bold solid lines. Again, we observe that when combined with and EOS, the liquid phase $G^{\text {ex }}$ are reduced compared to that directly from the LM. Furthermore, the value of $G^{\text {ex }}$ from PR + WS + COSMOSAC is significantly lower than that from PR + WS + COSMOSACres, resulting in a worsened accuracy in VLE prediction, especially at high temperatures. Together with the fact that the combinatorial $G^{\text {ex }}$ dominates at high temperatures, the SG combinatorial term appears to be inadequate for this system.

\section{Conclusion}

In this work we examined the combination of PR EOS and a predictive LM through the WS mixing rule, and compared it to the use of simple VDW one fluid mixing rule. We found that for nearly ideal systems, all three methods $(\mathrm{PR}+\mathrm{VDW}$, $\mathrm{PR}+\mathrm{WS}+\mathrm{COSMOSAC}$, and $\left.\mathrm{PR}+\mathrm{WS}+\mathrm{COSMOSAC}^{\text {res }}\right)$ perform equally well. For systems with specific interactions (such as H-bond), using the WS mixing rule with an accurate LM does provide decent VLE predictions over a large range of temperature and pressure. The combination of LM with an EOS tends to lower the liquid phase nonideality $\left(G^{\mathrm{ex}}\right)$ compare to the use of LM alone. At high temperatures the combinatorial term in the LM dominates and a good combinatorial model is necessary for an accurate phase equilibrium prediction.

The SG combinatorial model does not appear to be appropriate for the systems considered here. For highly nonideal mixtures at low temperatures, the residual term dominates and combinatorial term is negligible. At high temperatures when the combinatorial term dominates, the SG model provides erroneous excess Gibbs free energy. As a consequence, neglecting the SG term in the COSMO-SAC model has a positive effect in the VLE prediction in the composite approach for all conditions. Therefore, the PR + WS + COSMOSAC ${ }^{\text {res }}$ approach is the best among the three methods tested here under all conditions.

\section{Acknowledgements}

The author would like to thank the financial support from Grant NSC 95-2221-E-002-290 by the National Science Council of Taiwan and the computational facility in National Center for High-Performance Computing in Taiwan.

\section{References}

[1] S.I. Sandler, Chemical and Engineering Thermodynamics, 3rd ed., John Wiley \& Sons Inc., New York, 1999.

[2] J.M. Prausnitz, F.W. Tavares, AIChE J. 50 (2004) 739

[3] D. Peng, D.B. Robinson, Ind. Eng. Chem. Fundam. 15 (1976) 59.

[4] G. Soave, Chem. Eng. Sci. 27 (1972) 1197.

[5] H. Renon, J.M. Prausnitz, AIChE J. 14 (1968) 135.

[6] G.M. Wilson, J. Am. Chem. Soc. 86 (1964) 127.

[7] D.S. Abrams, J.M. Prausnitz, AIChE J. 21 (1975) 116.

[8] C. Boukouvalas, N. Spiliotis, P. Coutsikos, N. Tzouvaras, D. Tassios, Fluid Phase Equilib. 92 (1994) 75.

[9] M.M. Dahl S, AIChE J. 36 (1990) 1829.

[10] T. Holderbaum, A. Utzig, J. Gmehling, Fluid Phase Equilib. 63 (1991) 219.

[11] M.J. Huron, J. Vidal, Fluid Phase Equilib. 3 (1979) 255.

[12] M.L. Michelsen, Fluid Phase Equilib. 60 (1990) 213.

[13] J. Mollerup, Fluid Phase Equilib. 25 (1986) 323.

[14] H. Orbey, S.I. Sandler, Fluid Phase Equilib. 111 (1995) 53.

[15] D.S.H. Wong, S.I. Sandler, AIChE J. 38 (1992) 671.

[16] P.M. Mathias, H.C. Klotz, J.M. Prausnitz, Fluid Phase Equilib. 67 (1991) 31.

[17] J. Schwartzentruber, H. Renon, Fluid Phase Equilib. 67 (1991) 99.

[18] R. Sandoval, G. Wilczekvera, J.H. Vera, Fluid Phase Equilib. 52 (1989) 119.

[19] A.Z. Panagiotopoulos, R.C. Reid, Fluid Phase Equilib. 29 (1986) 525.

[20] Y. Adachi, H. Sugie, Fluid Phase Equilib. 28 (1986) 103.

[21] A.Z. Panagiotopoulos, R.C. Reid, 300 (1986) 571.

[22] D. Luedecke, J.M. Prausnitz, Fluid Phase Equilib. 22 (1985) 1.

[23] A. Klamt, J. Phys. Chem. 99 (1995) 2224.

[24] S.T. Lin, S.I. Sandler, Ind. Eng. Chem. Res. 41 (2002) 899.

[25] G.N. Escobedo-Alvarado, S.I. Sandler, A.M. Scurto, J. Supercrit. Fluids 21 (2001) 123.

[26] M.B. Shiflett, S.I. Sandler, Fluid Phase Equilib. 147 (1998) 145.

[27] D. Constantinescu, A. Klamt, D. Geana, Fluid Phase Equilib. 231 (2005) 231.

[28] Y. Shimoyama, Y. Iwai, S. Takada, Y. Arai, T. Tsuji, T. Hiaki, Fluid Phase Equilib. 243 (2006) 183.

[29] $\mathrm{DMol}^{3}$, Cerius ${ }^{2}$, Molecular Simulations Inc., San Diego, 1999.

[30] J.G. Gmehling, Vapor-liquid equilibrium data collection, 1977 and onward.

[31] S. Ohe, Vapor-Liquid Equilibrium Data at High Pressure, Elsevier, Kodansha, Tokyo, 1990

[32] D.E. Matschke, G. Thodos, J. Chem. Eng. Data 7 (1962) 232.

[33] J. Shim, J.P. Kohn, J. Chem. Eng. Data 7 (1) (1962) 3.

[34] P. Marteau, J. Obriot, A. Barreau, V. RuffierMeray, E. Behar, Fluid Phase Equilib. 129 (1997) 285.

[35] Y. Kim, W. Bae, H. Kim, J. Chem. Eng. Data 50 (2005) 1520.

[36] J. Seo, J. Lee, H. Kim, Fluid Phase Equilib. 172 (2000) 211.

[37] S. Ohe, Vapor-Liquid Equilibrium Data, Elsevier, Kodansha, Tokyo, 1989.

[38] F. Barr-David, B.F. Dodge, J. Chem. Eng. Data 4 (1959) 107. 